\title{
Antimycobacterial and antimalarial activities of endophytic fungi associated with the ancient and narrowly endemic neotropical plant Vellozia gigantea from Brazil
}

\author{
Mariana C Ferreira', Charles L Cantrell'2, David E Wedge², Vívian N Gonçalves', \\ Melissa R Jacob ${ }^{3}$, Shabana Khan ${ }^{3}$, Carlos A Rosa', Luiz H Rosa ${ }^{1 /+}$
}

'Universidade Federal de Minas Gerais, Departamento de Microbiologia, Belo Horizonte, MG, Brasil
${ }^{2}$ United States Department of Agriculture, Natural Products Utilization Research Unit, Mississippi, USA
${ }^{3}$ University of Mississippi, National Center for Natural Products Research, Mississippi, USA

BACKGROUND Endophytic fungi, present mainly in the Ascomycota and Basidiomycota phyla, are associated with different plants and represent important producers of bioactive natural products. Brazil has a rich biodiversity of plant species, including those reported as being endemic. Among the endemic Brazilian plant species, Vellozia gigantea (Velloziaceae) is threatened by extinction and is a promising target to recover endophytic fungi.

OBJECTIVE The present study focused on bioprospecting of bioactive compounds of the endophytic fungi associated with $V$. gigantea, an endemic, ancient, and endangered plant species that occurs only in the rupestrian grasslands of Brazil.

METHODS The capability of 285 fungal isolates to produce antimicrobial and antimalarial activities was examined. Fungi were grown at solid-state fermentation to recover their crude extracts in dichloromethane. Bioactive extracts were analysed by chromatographic fractionation and NMR and displayed compounds with antimicrobial, antimycobacterial, and antimalarial activities.

FINDINGS Five fungi produced antimicrobial and antimalarial compounds. Extracts of Diaporthe miriciae showed antifungal, antibacterial, and antimalarial activities; Trichoderma effusum displayed selective antibacterial activity against methicillinresistant Staphylococcus aureus and Mycobacterium intracellulare; and three Penicillium species showed antibacterial activity. D. miriciae extract contained highly functionalised secondary metabolites, yielding the compound epoxycytochalasin $\mathrm{H}$ with high antimalarial activity against the chloroquine-resistant strain of Plasmodium falciparum, with an $\mathrm{IC}_{50}$ approximately 3.5 -fold lower than that with chloroquine.

MAIN CONCLUSION Our results indicate that $V$. gigantea may represent a microhabitat repository hotspot of potential fungi producers of bioactive compounds and suggest that endophytic fungal communities might be an important biological component contributing to the fitness of the plants living in the rupestrian grassland.

Key words: ancient plant - antimicrobial - fungi - malaria - natural products

Endophytic fungi are an important source of bioactive metabolites, with a wide range of different biological activities (Strobel et al. 2004, Rosa et al. 2010). According to Strobel et al. (2004), plants from unique environmental settings, endemic species, and those with unusual longevity can be interesting targets for the recovery of unique endophytic species able to produce bioactive compounds. According to Rosa et al. (2011), endophytic fungi include a high diversity of species, mainly in the Ascomycota and Basidiomycota phyla and their anamorphs, associated with different plants around the world and representing important producers of bioactive natural products.

Brazil has a rich biodiversity of plant species, including those reported as being endemic. Among the endemic plants of the rupestrian grasslands, those of

doi: 10.1590/0074-02760170144

Financial support: FAPEMIG (0050-13), CAPES (23038.003478/2013-92), FINEP (2084/07), NIH, NIAID, Division of AIDS (grant no AI 270940, USDA Agricultural Research Service Specific Cooperative Agreement (no 58-6408-1-603).

+ Corresponding author: Ihrosa@icb.ufmg.br

Received 11 April 2017

Accepted 11 May 2017
Velloziaceae occur at a high frequency and contain approximately 240 predominately neotropical species and several other species (Menezes et al. 1994, Lousada et al. 2011). Vellozia, known locally as 'canela-de-ema', is the largest genus in the family and includes approximately 105 species (Menezes et al. 1994). Vellozia gigantea $\mathrm{N}$. L. Menezes \& Mello-Silva (Velloziaceae), a recently described species, is threatened by extinction (Lousada et al. 2011). According to Alves (1994), the dracenoid species of Vellozia, like $V$. gigantea, may represent an ancient plant that could be hundreds of thousands of years old. In the present study, we focused on exploring the tropical endophytic fungi of $V$. gigantea as a source of antimicrobial and antimalarial compounds for use as prototype molecules to treat neglected tropical diseases.

\section{MATERIALS AND METHODS}

Isolation of endophytic fungi - The endophytic fungi were recovered from leaves and adventitious roots of the endemic neotropical plant $V$. gigantea from the Brazilian rupestrian grasslands (Ferreira et al. 2017). The fungi were obtained from the Culture Collection of Microorganisms and Cells of the Federal University of Minas Gerais to cultivate and produce their crude extracts. 
Fungal cultivation and preparation of extracts for biological assays - All fungal isolates were cultivated according to protocols established by Rosa et al. (2013). In brief, a 5-mm-diameter plug of each isolate was placed on $20 \mathrm{~mL}$ of PDA medium at the centre of the Petri dishes $(90 \mathrm{~mm}$ diameter) and cultured for 15 days at $25 \pm$ $2^{\circ} \mathrm{C}$ (enough time for fungi to produce secondary metabolites). These fungal cultures were lyophilised for 72 $\mathrm{h}$, cut into small pieces, and transferred to $50-\mathrm{mL}$ glass centrifuge tubes, to which $50 \mathrm{~mL}$ of dichloromethane (DCM; Fisher Scientific, USA) was then added. After 72 $h$ at room temperature, the organic phase was filtered, and the solvent was removed under rotary evaporation at $40^{\circ} \mathrm{C}$. An aliquot of each dried extract was dissolved in dimethyl sulphoxide (Merck, USA) to prepare a 100-mg $\mathrm{mL}^{-1}$ stock solution, which was stored at $-20^{\circ} \mathrm{C}$. Sterile PDA medium was extracted under the same procedure and used as the control in the screening tests.

Assays for antimicrobial activity - Susceptibility testing of the fungal extracts, fractions and compounds from purification of extracts were performed using $\mathrm{Can}$ dida albicans ATCC 90028, C. glabrata ATCC 90030, C. krusei ATCC 6258, Cryptococcus neoformans ATCC 90113, Aspergillus fumigatus ATCC 204305, Staphylococcus aureus ATCC 29213, methicillin-resistant S. aureus ATCC 33591 (MRS), Escherichia coli ATCC 35218, Pseudomonas aeruginosa ATCC 27853, and Mycobacterium intracellulare ATCC 23068. All microorganisms were obtained from the American Type Culture Collection (Manassas, VA) and tested using versions of the CLSI (formerly NCCLS) methods (CLSI 2002a, b, 2003, 2006). A bioassay test on $M$. intracellulare was performed as previously described with modifications (Franzblau et al. 1998). Samples were serially diluted in $20 \% \mathrm{DMSO} /$ saline and transferred in duplicate to 96 -well flat bottom microplates. Microbial inocula $\left(1-2 \times 10^{8}\right.$ bacterial cells $\mathrm{mL}^{-1}$ ) were prepared by correcting the $\mathrm{OD}_{630}$ of microbe suspensions in incubation broth to create final target inocula. Ciprofloxacin (ICN Biomedicals, Ohio) at $1 \mu \mathrm{g}$ $\mathrm{mL}^{-1}$ for bacteria and amphotericin B (ICN Biomedicals, Ohio) at $5 \mu \mathrm{g} \mathrm{mL}^{-1}$ for fungi were included in each assay as positive controls. All assayed microorganisms were read at either $530 \mathrm{~nm}$ using the Biotek Powerwave XS plate reader (Bio-Tek Instruments, Vermont) or 544ex $1590 \mathrm{em}$ (M. intracellulare, A. fumigatus) using the Polarstar Galaxy Plate Reader (BMG LabTechnologies, Germany) prior to and after incubation. Percent growth was plotted versus test concentration to determine the $\mathrm{IC}_{50}$.

Assay for screening antimalarial activity and cytotoxicity - The antimalarial activity was determined against strains of Plasmodium falciparum chloroquine sensitive (D6) and chloroquine resistant (W2) by measuring plasmodial LDH activity (Makler \& Hinrichs 1993). A suspension of red blood cells infected with the D6 or W2 strain of P. falciparum $(200 \mu \mathrm{L}$, with $2 \%$ parasitaemia and $2 \%$ haematocrit in RPMI 1640 medium supplemented with $10 \%$ human serum and $60 \mu \mathrm{g} \mathrm{mL}^{-1}$ Amikacin) was added to the wells of a 96-well plate containing 10 $\mu \mathrm{L}$ of serially diluted samples (fungal extracts, fractions or pure compounds). The plate was incubated at $37^{\circ} \mathrm{C}$ for
$72 \mathrm{~h}$ in a modular incubation chamber with $90 \% \mathrm{~N}_{2}, 5 \%$ $\mathrm{O}_{2}$, and $5 \% \mathrm{CO}_{2}$. Parasitic LDH activity was determined by mixing $20 \mu \mathrm{L}$ of the incubation mixture with $100 \mu \mathrm{L}$ of Malstat reagent and incubating at room temperature for 30 min. Twenty microlitres of a 1:1 mixture of NBT/PES (Sigma, St. Louis, MO) was added and the plate incubated in the dark for $1 \mathrm{~h}$. The reaction was stopped by adding $100 \mu \mathrm{L}$ of a $5 \%$ acetic acid solution, and the absorbance was read at $650 \mathrm{~nm}$. Chloroquine at $10.33 \mathrm{ng} \mathrm{mL}^{-1}$ for D6, $137.65 \mathrm{ng} \mathrm{mL}^{-1}$ for $\mathrm{W} 2$; and Artemisinin at $2.87 \mathrm{ng}$ $\mathrm{mL}^{-1}$ for D6 and $3.21 \mathrm{ng} \mathrm{mL}^{-1}$ for $\mathrm{W} 2$ were included as the drug controls. $\mathrm{IC}_{50}$ values were computed from the dose response curves of growth inhibition using XLfit 4.2.0. The in vitro cytotoxicity to mammalian cell samples was tested to determine the selectivity index of the antimalarial activity. The assay was performed in 96-well tissue culture-treated plates. Vero cells (monkey kidney fibroblasts) were seeded to the wells of 96-well plate at a density of 25,000 cells well ${ }^{-1}$ and grown for $24 \mathrm{~h}$. Samples at different concentrations were added and the cells were incubated for $48 \mathrm{~h}$. Cell viability was determined by the Neutral Red method at $40 \mu \mathrm{g} \mathrm{mL}^{-1}$ (Borenfreund et al. 1990). Absorbance was recorded at $540 \mathrm{~nm}$ with an enzyme-linked immune assay-type microtiter plate reader. $\mathrm{IC}_{50}$ values were obtained from dose response curves.

NMR spectroscopy - Bioactive fungal extracts, fractions and pure compounds were analysed by NMR spectroscopy on a Bruker UXNMR $500 \mathrm{MHz}$ spectrometer (Billerica, MA, USA). ${ }^{1} \mathrm{H}$ and ${ }^{13} \mathrm{C}$ NMR spectra recorded in DMSO-d ${ }_{6}$ using a standard ${ }^{1} \mathrm{H}$ NMR pulse program.

Crude extract preparation for bioassay-directed purification - Five-millimetre-diameter plugs of each fungal isolate were placed onto $20 \mathrm{~mL}$ of PDA medium at the centre of 350 Petri dishes $(90 \mathrm{~mm}$ diameter) and cultured for 15 days at $25 \pm 2^{\circ} \mathrm{C}$. The fungal cultures were lyophilised for $72 \mathrm{~h}$, cut into small pieces, and transferred to $50-\mathrm{mL}$ glass centrifuge tubes, to which $50 \mathrm{~mL}$ of DCM was then added. After $72 \mathrm{~h}$ at room temperature, the organic phase was filtered, and the solvent was removed under rotary evaporation at $40^{\circ} \mathrm{C}$. Initially, $1.149 \mathrm{~g}$ of Diaporthe miriciae UFMGCB 9720 extract was adsorbed to silica gel and applied to a silica gel chromatography column $(40-63 \mu \mathrm{m}, 40 \times$ $150 \mathrm{~mm}, 60 \AA$ ) in a Biotage XP-Sil system. The column was eluted at a flow rate of $40 \mathrm{~mL} \mathrm{~min}{ }^{-1}$ using hexane/ EtOAc mixtures with the following gradient: $100 \%$ hexane: $0 \%$ EtOAc to $0 \%$ hexane:100\% EtOAc over 3,024 $\mathrm{mL}$, and finishing with a $350-\mathrm{mL} \mathrm{MeOH}$ wash. The column eluate was collected in 27-mL fractions and, based on TLC similarities performed with the solvent hexane/ EtOAc, recombined into 9 fractions [(A) 1-16, $7.7 \mathrm{mg}$; (B) 17-20, $9.1 \mathrm{mg}$; (C) 21-29, $441.4 \mathrm{mg}$; (D) 30-40, 9.3 mg; (E) 41-43, $11.2 \mathrm{mg}$; (F) 44-55, $133.8 \mathrm{mg}$; (G) 5687, $46.2 \mathrm{mg}$; (H) 87-112, $12 \mathrm{mg}$; and (I) wash column, $479.3 \mathrm{mg}$ ]. Fractions $\mathrm{H}$ and I were selected for further investigation based on their activities in the antimalarial assays against $P$. falciparum. TLC and ${ }^{1} \mathrm{H}$ NMR analyses displayed the same chemical profile for fractions $\mathrm{H}$ and I. Fraction I was selected and adsorbed to silica gel and applied to the silica gel chromatography column. 
Elution of the column was performed using increasing polarity mixtures of hexane:isopropyl alcohol in a series of 4 linear steps as follows: (step 1) 100:0 to 80:20 over $2,400 \mathrm{~mL}$, (step 2) $80: 20$ to $50: 50$ over $1,200 \mathrm{~mL}$, (step 3) 50:50 to 0:100 over $152 \mathrm{~mL}$, and (step 4) 0:100 over 396 $\mathrm{mL}$. The column eluate was collected into $27-\mathrm{mL}$ portions and, based on TLC similarities performed with the solvent hexane/IPA, recombined into six fractions [(A') 1-54, $18.8 \mathrm{mg}$; (B') 55-59, $69.5 \mathrm{mg}$; (C') 60-70, $168.7 \mathrm{mg}$; (D') 71-77, $15.2 \mathrm{mg}$; and (E') 78-169, $23.5 \mathrm{mg}$. Fractions B' and C' were identified as epoxycytochalasin $H$ and selected for further investigation based on their activities in the antimalarial assays.

Identification of epoxycytochalasin $H-{ }^{13} \mathrm{C}$ NMR (120 MHz in DMSO-d ${ }_{6}$ ) $\delta 174.27$ (C-1), 170.04 (C-21Ac), 138.67 (C-20), 137.11 (C-1'), 134.13 (C-14), 129.58 (C-2' and $\left.6^{\prime}\right), 128.49$ (C-13), 128.36 (C-3' and 5'), $126.52(\mathrm{C}-$ $\left.4^{\prime}\right), 124.49$ (C-19), 75.39 (C-21), 72.22 (C-18), 62.34 (C-7), 56.74 (C-6), 53.68 (C-17), 53.52 (C-9), 53.23 (C-3), 48.52 (C-4), 44.80 (C-10), 44.67 (C-8), 42.56 (C-15), 35.89 (C-5), 30.45 (C-23), 27.58 (C-16), 26.05 (C-22), 20.44 (C-21Ac),
19.21 (C-12), 11.95 (C-11). ${ }^{13} \mathrm{C}$ NMR data (Supplementary data, Figure) for fraction B' indicated complete agreement with a previous report (Izawa et al. 1989) providing structural confirmation as epoxycytochalasin $\mathrm{H}$.

\section{RESULTS}

Among all fungal extracts screened, five displayed at least one biological activity against the different targets. Among them, the extracts of D. miriciae UFMGCB 9720 showed antifungal and antibacterial activities, with MIC ranging from 9.98 to $148.79 \mu \mathrm{g} \mathrm{mL}^{-1}$, and antimalarial activities with $94 \%$ inhibition (Table I). Trichoderma effusum displayed selective antibacterial activity against methicillin-resistant $S$. aureus and $M$. intracellulare. Three Penicillium species (P. herquei, P. adametzii, and $P$. quebecense) showed antibacterial activity.

All bioactive extracts were examined using ${ }^{1} \mathrm{H}$ NMR analysis for the presence of secondary metabolites with interesting chemical shifts. The extracts of T. effusum, $P$. herquei, $P$. adametzii, and Diaporthe sp. showed only the presence of fatty acids and, for this reason, they were not

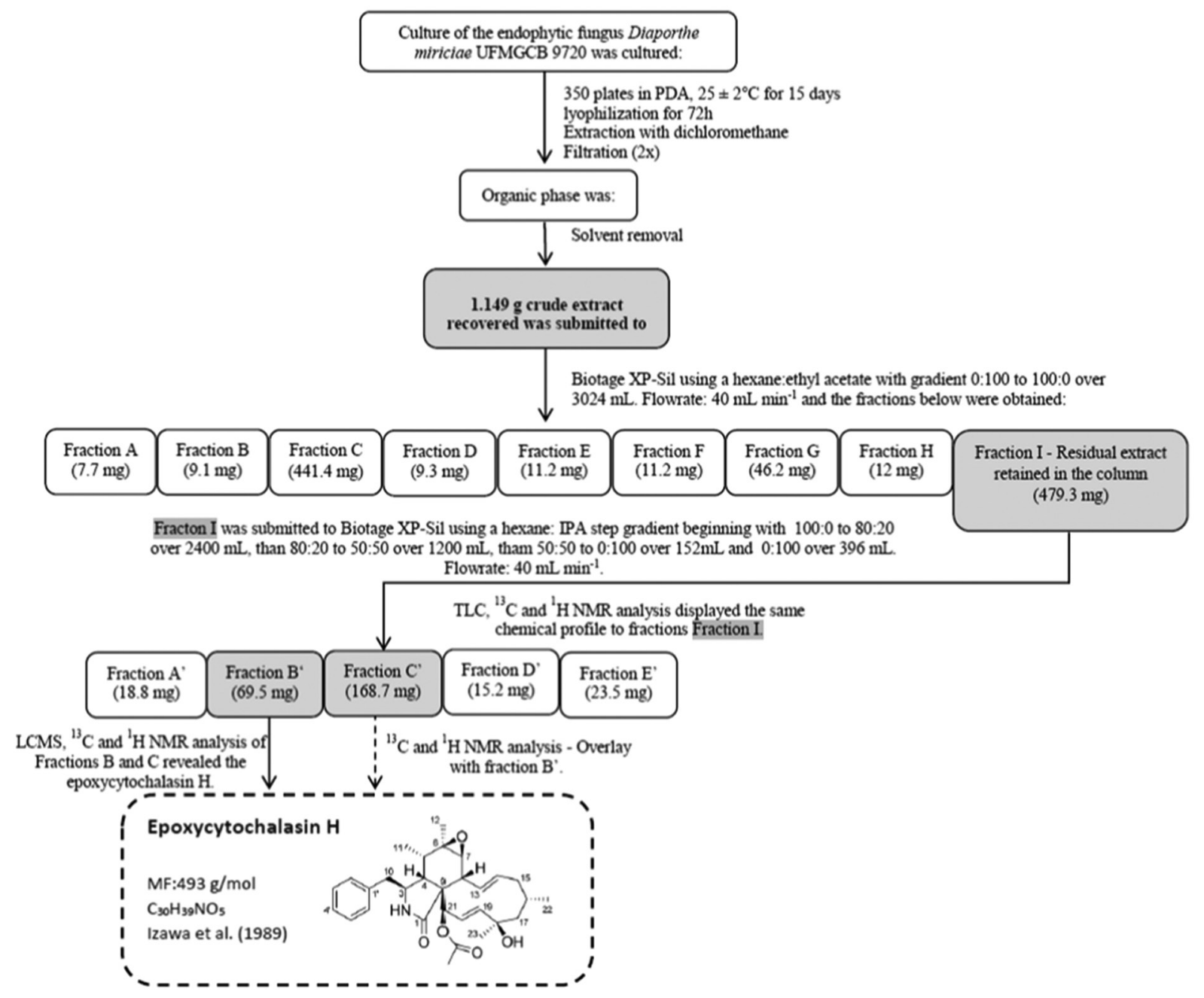

Flowcharts illustrating the processes of chemical isolation of the compound epoxycytochalasin $\mathrm{H}$ obtained from the endophytic fungus Diaporthe miriciae UFMGCB 9720. 


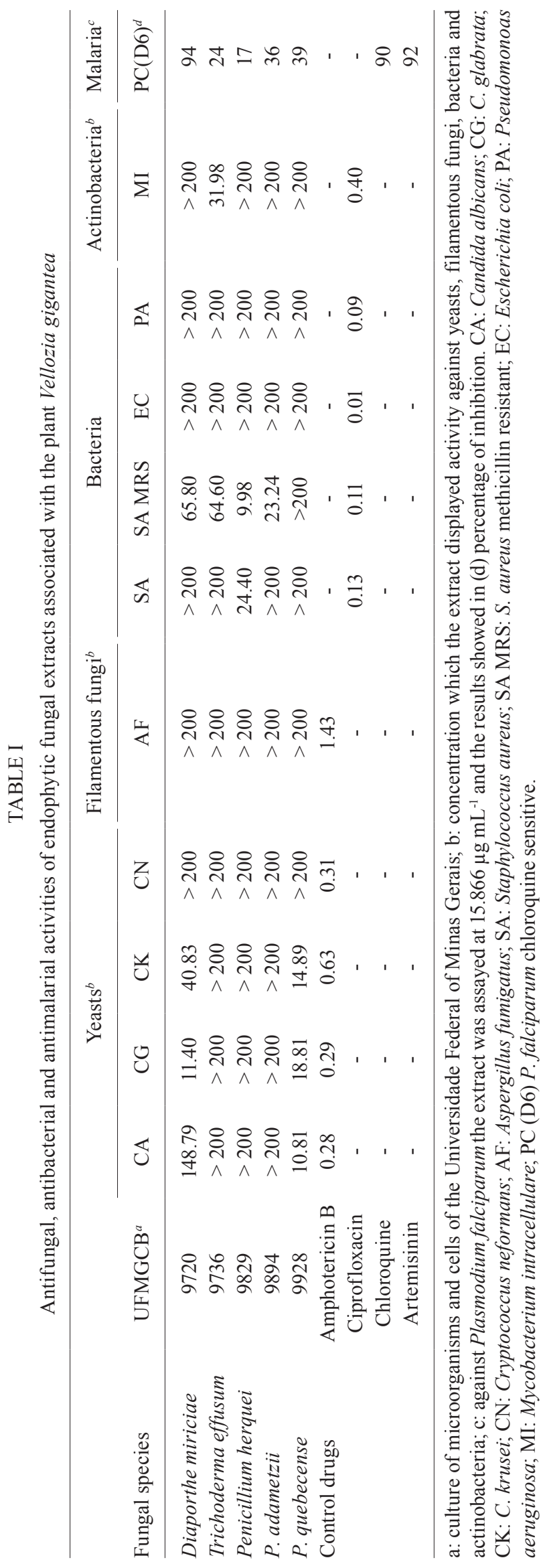

subjected only to bioassay-directed purification. In contrast, the $D$. miriciae UFMGCB 9720 extract showed the presence of highly functionalised secondary metabolites because of the presence of protons in the aromatic and olefinic regions. Detection of such compounds is clear from NMR chemical shifts, indicative of olefinic protons, aromatic protons, oxygenated methylene protons, and olefinic methyl protons. Therefore, the extract of $D$. miriciae was fractioned, yielding $238.2 \mathrm{mg}$ of the compound epoxycytochalasin $\mathrm{H}$ (Figure), which displayed high antimalarial activity against both chloroquine-sensitive and chloroquine-resistant strains of $P$. falciparum, with $\mathrm{IC}_{50}$ values of 52 and $39 \mathrm{ng} \mathrm{mL}^{-1}$, respectively, without any cytotoxicity towards mammalian kidney (Vero) cells (Table II). The $\mathrm{IC}_{50}$ of epoxycytochalasin $\mathrm{H}$ in the chloroquine-resistant strain was approximately 3.5 -fold lower than that of the control drug chloroquine (Table II).

\section{DISCUSSION}

Diaporthe was the most abundant genus of endophytes recovered associated with $V$. gigantea. Additionally, the extract of D. miriciae UFMGCB 9720 showed antifungal, antibacterial, and antimalarial activities. The genera Diaporthe and Phomopsis form an anamorph/teleomorph complex, which is a known producer of different bioactive compounds (Agusta et al. 2006, Carvalho et al. 2012, Silva-Hughes et al. 2015). According to Thompson et al. (2015), Diaporthe species are known to be saprobic and pathogenic fungi but have also been reported as endophytes in a wide range of host plants. D. miriciae is a new species recently described by Thompson et al. (2015); it was obtained from the plant species Glycine max, Helianthus annuus, and Vigna radiata in Australia. According to Thompson et al. (2015), D. miriciae forms clusters of Diaporthe sojae, a pathogen of Glycine species, suggesting that it may also be a pathogen.

The endophyte $D$. miriciae UFMGCB 9720 produced epoxycytochalasin $\mathrm{H}$, which displayed high antimalarial activity against chloroquine-resistant $P$. falciparum. The cytochalasins are structurally complex secondary metabolites with more than 80 molecules described and have been isolated from fungi of the genera Aspergillus, Diaporthe/Phomopsis, Penicillium, Zygosporium, Chaetomium, Phoma, Xylaria, Hypoxylon, and Rhinocladiella (Dagne et al. 1994, Wagenaar et al. 2000, Zhang et al. 2012, 2014). Cytochalasins are a class of metabolites produced by fungi with antimicrobial, antitumour, anti-HIV, and herbicidal activities (Cimmino et al. 2008, Lin et al. 2009, Xu et al. 2009). Epoxycytochalasin H is produced by the soybean pathogen Phomopsis sojae (Cole et al. 1982) and by a Phoma sp. obtained from a soil sample (Kakeya et al. 1997). To the best of our knowledge, a unique activity of epoxycytochalasin $\mathrm{H}$ is its ability to function as a cell-cycle inhibitor in mammals (Kakeya et al. 1997).

In conclusion, our results indicate that $V$. gigantea shelters cryptic fungal species able to produce bioactive compounds in its tissues. According to Compant et al. (2016), the interaction with endophytes may be beneficial to the plant's fitness because recent studies of plantsoil-microbe interactions revealed the potential of some endophytic fungi as promising sources of secondary me- 
TABLE II

Antimalarial activity of the compound epoxycytochalasin $\mathrm{H}$ isolated from the endophytic fungus Diaporthe miriciae UFMGCB 9720

\begin{tabular}{lccccc}
\hline & \multicolumn{4}{c}{ Plasmodium falciparum } \\
\cline { 2 - 6 } Compound & $\mathrm{D}^{a}-\mathrm{IC}_{50}$ & $\mathrm{D}^{a}-\mathrm{SI}$ & $\mathrm{W}^{b}-\mathrm{IC}_{50}$ & $\mathrm{~W}^{b}-\mathrm{SI}$ & $\mathrm{VERO}^{c} \mathrm{IC}_{50}$ \\
\hline Epoxycytochalasin H & 51.70 & $>92.10$ & 39.40 & $>120.70$ & $>4760$ \\
Chloroquine $^{\mathrm{d}}$ & 10.33 & $>23$ & 137.65 & $>1.70$ & $>238$ \\
Artemisinin $^{\mathrm{d}}$ & 2.87 & $>83$ & 3.21 & $>74$ & $>238$ \\
\hline
\end{tabular}

a: Plasmodium falciparum chloroquine sensitive (D6); b: P. falciparum chloroquine resistant (W2); c: Cell VERO; d: control drug; $\mathrm{IC}_{50}$ : inhibitory concentration of $50 \%$; SI: selectivity index. Values calculated at $\mathrm{ng} \mathrm{mL}^{-1}$.

tabolites for use in agriculture and medicine. Thus, epoxicitocalasin $\mathrm{H}$, with activity against $P$. falciparum reported for the first time, can be used as a prototype molecule to study antimalarial substances. Our results suggest that endophytic fungal communities may be an important biological component contributing to the fitness of the plants living in the rupestrian grassland and that those plants may represent a microhabitat repository hotspot of potential fungi producers of bioactive compounds.

\section{ACKNOWLEDGEMENTS}

To Solomon Green III, Ms JL Robertson, Mrs R Pace, and Mrs Amber Reichley for technical assistance.

\section{AUTHORS' CONTRIBUTION}

MCF collected the plants, isolated the endophytes, produced and assayed the extract and isolated the bioactive compounds; CLC isolated and identified the bioactive compounds; DEW was responsible for the biological activities; VNG produced and assayed the extract and isolated the bioactive compounds; MRJ was responsible for the antibacterial assay; SK was responsible for the biological assays; CAR isolated the fungi; LHR collected the plants, isolated the endophytes and produced the extract.

\section{REFERENCES}

Agusta A, Ohashi K, Shibuya H. Bisanthraquinone metabolites produced by the endophytic fungus Diaporthe sp. Chem Pharm Bull. 2006; 54(4): 579-82.

Alves RJV. Morphological age determination and longevity in some Vellozia populations in Brazil. Folia Geobot. 1994; 29(1): 55-9.

Borenfreund E, Babich H, Martin-Alguacil N. Rapid chemosensitivity assay with human normal and tumor cells in vitro. In Vitro Cell Dev-Pl. 1990; 26(11): 1030-4.

Carvalho CR, Gonçalves VN, Pereira CB, Johann S. The diversity, antimicrobial and anticancer activity of endophytic fungi associated with the medicinal plant Stryphnodendron adstringens (Mart.) Coville (Fabaceae) from the Brazilian savannah. Symbiosis. 2012; 57(2): 95-107.

Cimmino A, Andolfi A, Berestetskiy A, Evidente A. Production of phytotoxins by Phoma exigua var. exigua, a potential mycoherbicide against perennial thistles. J Agric Food Chem. 2008; 56(15): 6304-9.

CLSI - Clinical and Laboratory Standards Institute. Methods for dilution antimicrobial susceptibility tests for bacteria that grow aerobically; approved standard - Seventh edition. Clinical and Laboratory Standards Institute document M7-A7 [ISBN 1-56238587-9]. Wayne: CLSI; 2006; 26(2): 49 pp.
CLSI - Clinical and Laboratory Standards Institute. Reference method for broth dilution antifungal susceptibility testing of yeasts; approved standard M27-A2. Clinical and Laboratory Standards Institute document M27-A2. Wayne: CLSI; 2002a; 22(1): 15 pp.

CLSI - Clinical and Laboratory Standards Institute. Reference method for broth dilution antifungal susceptibility testing of filamentous fungi; approved standard M38-A. Clinical and Laboratory Standards Institute document M38-A. Wayne: CLSI; 2002b; 22(1): 16 pp.

CLSI - Clinical and Laboratory Standards Institute. Susceptibility testing of mycobacteria, nocardia, and other aerobic actinomycetes; Tentative Standard; approved standard M24-A. Clinical and Laboratory Standards Institute document M24-A. Wayne: CLSI; 2003; 23(1): 18 pp.

Cole RJ, Wilson DM, Harper JL, Cox RH. Isolation and identification of two new [11] cytochalasins from Phomopsis sojae. J Agric Food Chem. 1982; 30(1): 301-4.

Compant S, Saikkonen K, Mitter B, Campisano A. Editorial special issue: soil, plants and endophytes. Plant Soil. 2016; 405(2): 1-11.

Dagne E, Gunatilaka AAL, Asmellash S, Abate D. Two new cytotoxic cytochalasins from Xylaria obovata. Tetrahedron. 1994; 50(19): 5615-20.

Ferreira MC, Cantrell CL, Wedge DE, Gonçalves VN. Diversity of the endophytic fungi associated with the ancient and narrowly endemic neotropical plant Vellozia gigantea from the endangered Brazilian rupestrian grasslands. Biochem Syst Ecol. 2017; 71: 163-9.

Franzblau SG, Witzig RS, McLaughlin J, Torres P. Rapid, low-technology MIC determination with clinical Mycobacterium tuberculosis isolates by using the microplate alamar blue assay. J Clin Microbiol. 1998; 36(2): 362-6.

Izawa Y, Hirose T, Shimizu T, Koyama K. Six new 10-5 pheynl-[11] cytochalasans, cytochalasins N-S from Phomopsis sp. Tetrahedron. 1989; 45(8): 2323-35.

Kakeya H, Morishita M, Onozawa C, Usami R. RKS-1778, a new mammalian cell-cycle inhibitor and a key intermediate of the cytochalasin group. J Nat Prod. 1997; 60(7): 669-72.

Lin Z, Zhang G, Zhu T, Liu R. Bioactive cytochalasins from Aspergillus flavipes, an endophytic fungus associated with the mangrove plant Acanthus ilicifolius. Helv Chim Acta. 2009; 92(8): 1538-44.

Lousada JM, Borb E, Ribeiro KT, Ribeiro LC. Genetic structure and variability of the endemic and vulnerable Vellozia gigantea (Velloziaceae) associated with the landscape in the Espinhaço Range, in southeastern Brazil: implications for conservation. Genetica. 2011; 139(4): 431-40.

Makler MT, Hinrichs DJ. Measurement of the lactate dehydrogenase activity of Plasmodium falciparum as an assessment of parasitemia. J Trop Med Hyg. 1993; 48(2): 205-10. 
Menezes NL, Mello-Silva R, Mayo SJ. Cladistic analysis of the Velloziaceae. Kew Bull. 1994; 49(1): 71-92.

Rosa LH, Gonçalves VN, Caligiorne RB, Alves TMA. Leishmanicidal, trypanocidal, and cytotoxic activities of endophytic fungi associated with bioactive plants in Brazil. Braz J Microbiol. 2010; 41(2): 114-22.

Rosa LH, Queiroz SCN, Moraes RM, Wang X. Coniochaeta ligniaria: antifungal activity of the cryptic endophytic fungus associated with autotrophic tissue cultures of the medicinal plant Smallanthus sonchifolius (Asteraceae). Symbiosis. 2013; 60(3): 133-42.

Rosa LH, Vieira MLA, Cota BB, Johann S. Endophytic fungi of tropical forests: a promising source of bioactive prototype molecules for the treatment of neglected diseases. In: Ekinci D, editor. Drug development - a case study based insight into modern strategies. Croatia: Intech; 2011. p. 1-18.

Silva-Hughes AF, Wedge DE, Cantrell CL, Carvalho CR. Diversity and antifungal activity of the endophytic fungi associated with the native medicinal cactus Opuntia humifusa (Cactaceae) from the United State. Microbiol Res. 2015; 175(1): 67-77.
Strobel G, Daisy B, Castillo U, Harper J. Natural products from endophytic microorganisms. J Nat Prod. 2004; 67(2): 257-68.

Thompson SM, Tan YP, Shivas RG, Neate SM. Green and brown bridges between weeds and crops reveal novel Diaporthe species in Australia. Persoonia. 2015; 35(1): 39-49.

Wagenaar MM, Corwin J, Strobel G, Clardy J. Three new cytochalasins produced by an endophytic fungus in the genus Rhinocladiella. J Nat Prod. 2000; 63(12): 1692-5.

Xu S, Ge HM, Song YC, Shen Y. Cytotoxic cytochalasin metabolites of endophytic Endothia gyrosa. Chem Biodivers. 2009; 6(5): 739-45.

Zhang Q, Li HQ, Zong SC, Gao JM. Chemical and bioactive diversities of the genus Chaetomium secondary metabolites. Mini-Rev Med Chem. 2012; 12(2): 127-48.

Zhang Q, Xiao J, Sun QQ, Qin JC. Characterization of cytochalasins from the endophytic Xylaria sp. and their biological functions. J Agric Food Chem. 2014; 62(45): 10962-9. 